\title{
The role of a purine-specific nucleoside hydrolase in spore germination of Bacillus thuringiensis
}

\author{
Correspondence \\ Gang Liu \\ liug@sun.im.ac.cn \\ Huarong Tan \\ tanhr@im.ac.cn
}

Received 30 October 2007

Revised 4 February 2008

Accepted 6 February 2008

\section{Liang Liang, ${ }^{1,2}$ Xihong He, ${ }^{1,2}$ Gang Liu ${ }^{1}$ and Huarong Tan ${ }^{1}$}

\author{
${ }^{1}$ State Key Laboratory of Microbial Resources, Institute of Microbiology, Chinese Academy of \\ Sciences, Beijing 100101, PR China \\ ${ }^{2}$ Graduate School of Chinese Academy of Sciences, Beijing 100039, PR China
}

\begin{abstract}
A homologous gene $($ iun $\mathrm{H})$ of a putative nucleoside hydrolase $(\mathrm{NH})$, which had been identified from the exosporia of Bacillus cereus and Bacillus anthracis spores, was cloned from Bacillus thuringiensis subsp. kurstaki. Disruption of iunH did not affect the vegetative growth and sporulation of Bacillus thuringiensis, but promoted both inosine- and adenosine-induced spore germination. The inosine- or adenosine-induced germination rate decreased when the wild-type iunH gene was overexpressed in Bacillus thuringiensis. The iunH gene product was characterized as a purine-specific $\mathrm{NH}$. The kinetic parameters of lunH with inosine as substrate were $K_{\mathrm{m}}=399 \pm 115 \mu \mathrm{M}, k_{\mathrm{cat}}=48.9 \pm 8.5 \mathrm{~s}^{-1}$ and $k_{\mathrm{cat}} / K_{\mathrm{m}}=1.23 \times 10^{5} \mathrm{M}^{-1} \mathrm{~s}^{-1}$. The optimal $\mathrm{pH}$ and temperature for lunH were found to be $\mathrm{pH} 6$ and $80{ }^{\circ} \mathrm{C}$. Meanwhile, the specific activity of inosine hydrolase in intact spores of the wild-type strain with inosine as substrate was

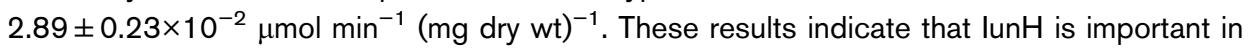
moderating inosine- or adenosine-induced germination of Bacillus thuringiensis spores.
\end{abstract}

\section{INTRODUCTION}

Enzymes with nucleoside hydrolase $(\mathrm{NH})$ activity are widely distributed in both prokaryotes and eukaryotes (Giabbai \& Degano, 2004). Non-specific NHs, the most well-known of which is the inosine/uridine-preferring $\mathrm{NH}$ (IU-NH) from Crithidia fasciculata, hydrolyse both purine and pyrimidine nucleosides (Parkin et al., 1991). Purinespecific $\mathrm{NHs}$ (inosine/adenosine/guanosine-preferring NHs] display strict specificity (Parkin, 1996; Pelle et al., 1998; Versees et al., 2001). NHs are crucial in the salvage pathway of protozoan parasites which are deficient in the pathway for de novo synthesis of purine nucleotides (Gopaul et al., 1996). Non-parasitic NHs, for example the salivary purine nucleosidase of the mosquito Aedes aegypti, may be involved in the degradation of modified nucleosides found in tRNA (Giabbai \& Degano, 2004; Ribeiro \& Valenzuela, 2003), but apart from this, no clear role for non-parasitic $\mathrm{NHs}$, including the $\mathrm{NH}$ identified from the exosporia of Bacillus anthracis and Bacillus cereus, has been reported so far (Giabbai \& Degano, 2004; Redmond et al., 2004; Ribeiro \& Valenzuela, 2003; Todd et al., 2003).

Bacillus thuringiensis, $B$. anthracis and $B$. cereus are members of the Gram-positive endospore-forming $B$. cereus group. They could be classified as one species on

Abbreviation: $\mathrm{NH}$, nucleoside hydrolase.

The GenBank/EMBL/DDBJ accession number for the nucleotide sequence of iunH is EU072023. the basis of genetic evidence, even though they demonstrate widely different phenotypes and pathological effects (Helgason et al., 2000). As a natural pesticide, commercial formulations of $B$. thuringiensis containing separate entities of crystals and spores has become the leading biological insecticide used to control agricultural pests (Crickmore, 2006; Liu et al., 1998). B. anthracis is the cause of the acute and often lethal disease anthrax, and $B$. cereus is a ubiquitous soil bacterium and opportunistic human pathogen (Helgason et al., 2000).

Under conditions of nutrient depletion at high cell density, vegetative cells of Bacillus species can transform into spores by a process called sporulation. The spore is metabolically dormant, and resistant to heat, radiation, desiccation, $\mathrm{pH}$ extremes and toxic chemicals. The dormant spore also monitors its environment, and when conditions become favourable again, the spore germinates and is converted back into a new vegetative cell (Setlow, 2003). In nature, spores probably germinate in response to nutrient germinants which are generally single amino acids, sugars or purine nucleosides (Setlow, 2003). Among nutrient germinants, the combination of L-alanine and inosine is the best germinant for most Bacillus spores. Inosine is an independent germinant for $B$. cereus and $B$. thuringiensis spores, and an important co-germinant in B. thuringiensis, B. cereus and B. anthracis spore germination (Foerster \& Foster, 1966; Hornstra et al., 2006).

The first event in nutrient-induced spore germination is probably the activation of the germinant receptors, which 
are located in the inner membrane of the spore (Hornstra et al., 2005). To activate the receptors, germinants must first penetrate the outer spore layers and access their corresponding receptors. The outermost layer of the $B$. cereus group spore is a loose-fitting, balloon-like structure known as an exosporium, which consists mainly of protein, polysaccharides, lipids and ash (Matz et al., 1970). Dozens of proteins, including a putative $\mathrm{NH}$, were identified from the exosporia of $B$. cereus and $B$. anthracis spores (Redmond et al., 2004; Steichen et al., 2003; Todd et al., 2003). Although the role of some proteins has been studied, most functions remain to be elucidated (Boydston et al., 2006; Ramarao \& Lereclus, 2005; Steichen et al., 2005; Yan et al., 2007).

Widespread distribution of $\mathrm{NH}$ raised the question of whether these genes indeed encode $\mathrm{NH}$ enzymes, and most importantly, what their role is, especially in non-parasitic organisms which could recycle nitrogenous bases via NPcatalysed phosphorolysis (Giabbai \& Degano, 2004). In this paper, we have characterized one non-parasitic, purinespecific NH from $B$. thuringiensis and demonstrated its role in moderating inosine- or adenosine-induced spore germination.

\section{METHODS}

Bacterial strains, plasmids and culture conditions. All the strains and plasmids used in this study are listed in Table 1. B. thuringiensis was routinely grown in Luria-Bertani (LB) medium at $30^{\circ} \mathrm{C}$ containing appropriate antibiotics $\left(25 \mu \mathrm{g}\right.$ erythromycin $\mathrm{ml}^{-1}$ or $10 \mu \mathrm{g}$ chloramphenicol $\mathrm{ml}^{-1}$ ). Casein hydrolysate/yeast-containing medium (CCY) was used for spore preparation (Stewart \& Halvorson, 1953). For subcloning, Escherichia coli $\mathrm{DH} 5 \alpha$ was grown at $37{ }^{\circ} \mathrm{C}$ in LB medium, containing ampicillin, erythromycin or chloramphenicol when necessary, for propagating plasmids.
DNA manipulations and transformation. B. thuringiensis genomic DNA was isolated as described previously (Gonzalez et al., 1981). Primers P1 (5'-GCTCTAGAGAACCGATAATACCAGC-3', XbaI site is underlined), P2 (5' - GGGGTACCGAAGTCGCCAATAAATAG-3', $K p n I$ site is underlined), P4 (5'-GGGGTACCGTCATAATAATCGTCTTCTTG-3', KpnI site is underlined) and P5 (5'-GCTCTAGACTAAATGATGAATTGGACC- ${ }^{\prime}, \quad X b a \mathrm{I}$ site is underlined) were designed according to the conserved sequence of the $B$. cereus group. The DNA fragment containing iunH was amplified from genomic DNA of B. thuringiensis subsp. kurstaki CGMCC 1.1752 with primers $\mathrm{P} 1$ and P2. After digestion with $X b a \mathrm{I}$ and $K p n \mathrm{I}$, the amplified DNA fragment was ligated into the corresponding sites of pKSV7 to generate pIC. Sequencing of the fragment was carried out by the SunBiotech Company (Beijing, PR China). Southern hybridization was carried out with probes labelled with a digoxigenin DNA labelling kit (Roche Biochemicals), according to the manufacturer's instructions. All PCRs were performed with Pfu DNA polymerase (TaKaRa) using standard conditions.

For B. thuringiensis electroporation, $80 \mu \mathrm{l}$ cell suspensions in $40 \%$ PEG were used per cuvette plus $1 \mu \mathrm{l}$ (about $1 \mu \mathrm{g}$ ) plasmid DNA (dissolved in distilled water) at $11 \mathrm{kV} \mathrm{cm}^{-1}, 1000 \Omega$ and $25 \mu \mathrm{F}$.

Construction of the iunH disruption mutant. To construct an iunH disruption mutant, a DNA fragment corresponding to the upstream region of $i u n H$ (extending from positions -1003 to +223 with respect to the iunH translation initiation site) was amplified with primers $\mathrm{P} 3$ (5'-ACGCGTCGACGCTGTCATCGGTCTACTC-3', SalI site is underlined) and $\mathrm{P} 4$, and a DNA fragment corresponding to the downstream region of $i u n H$ (extending from positions -306 to +846 with respect to the iunH stop codon) was amplified with primers P5 and P6 (5'CGGGATCCAATGATTGATTTTTATTATGAG-3', BamHI site is underlined) from $B$. thuringiensis. These PCR products were digested with SalI and BamHI, respectively. A $1256 \mathrm{bp}$ DNA fragment containing the erythromycin resistance gene $(\mathrm{erm})$ was amplified from pHT3101 with primers P7 (5'-ATAGGATCCAATAAGGGCGACACG$3^{\prime}$, BamHI site is underlined) and P8 (5'-ACGCGTCGACCCCTTAGAAGCAAACT-3', Sall site is underlined), and digested with SalI and BamHI. The digested PCR fragments were purified, mixed in equal amounts and ligated with T4 DNA ligase. The ligation mixture was used as a template to amplify the complete tripartite DNA fragment

Table 1. Bacterial strains and plasmids used in this study

\begin{tabular}{|c|c|c|}
\hline Strain or plasmid & Characteristics $^{\star}$ & Source or reference \\
\hline $\begin{array}{l}\text { B. thuringiensis subsp. kurstaki } \\
1.1752\end{array}$ & Wild-type strain for this study & CGMCC $\dagger$ \\
\hline \multicolumn{3}{|l|}{ E. coli } \\
\hline DH5 & 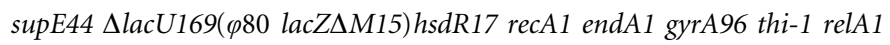 & Sambrook et al. (1989) \\
\hline BL21 (DE3) & hsdS gal ( $\lambda$ cIst857 indl sam7 nin5 lacUV5-T7 gene1) & Novagen \\
\hline \multicolumn{3}{|c|}{$x_{0}$} \\
\hline pBluescript KS & Cloning and subcloning vector & Stratagene \\
\hline pET28a & Expression vector in E. coli, $\mathrm{Km}^{\mathrm{r}}$ & Novagen \\
\hline pKSV7 & Integrative vector, $\mathrm{Ap}^{\mathrm{r}} \mathrm{Cm}^{\mathrm{r}}$ & Smith \& Youngman (1992) \\
\hline $\mathrm{pIC}$ & pKSV7 carrying iunH & This work \\
\hline pIE & pET28a carrying iunH & This work \\
\hline pID & pKSV7 : : iun $H:: \mathrm{Em}^{\mathrm{r}}$ & This work \\
\hline pHT3101 & Expression vector, $\mathrm{Ap}^{\mathrm{r}} \mathrm{Em}^{\mathrm{r}}$ & Lereclus et al. (1989) \\
\hline
\end{tabular}

${ }^{\star}$ Ap, Ampicillin; Cm, chloramphenicol; Em, erythromycin; Km, kanamycin.

$\nmid$ CGMCC, China General Microbiological Culture Collection Centre, Institute of Microbiology, Chinese Academy of Sciences, Beijing 100080, PR China. 
with primers $\mathrm{P} 4$ and $\mathrm{P} 5$. Then, the amplified $3.6 \mathrm{~kb}$ DNA fragment was digested with $\mathrm{Xba \textrm {I }}$ and $\mathrm{Kp} p \mathrm{I}$, and inserted into the corresponding sites of pKSV7 to give pID. Subsequently, pID was introduced into $B$. thuringiensis by electroporation, and cultured on LB plates containing erythromycin at $30{ }^{\circ} \mathrm{C}$ for 2 days. Transformants containing pID were selected and confirmed by plasmid isolation and digestion. One of the confirmed transformants was randomly selected and cultured overnight at $30{ }^{\circ} \mathrm{C}$ in LB broth without any antibiotic; then the culture was diluted and spread onto an LB plate containing erythromycin. After growing for about $10 \mathrm{~h}$ at $42{ }^{\circ} \mathrm{C}$, colonies were replicated on LB plates containing chloramphenicol. Then, the replicated plates and the original plates were cultured at $30{ }^{\circ} \mathrm{C}$. Chloramphenicol-sensitive and erythromycinresistant strains were selected. Subsequently, the disruption mutant was confirmed by PCR analysis and Southern hybridization.

For complementation experiments, plasmid pIC, containing iunH and its putative promoter region, was transformed into the iun $H$ disruption mutant by electroporation.

Spore preparation and germination analysis. To prepare spores, B. thuringiensis was grown in CCY medium at $30{ }^{\circ} \mathrm{C}$ for $48 \mathrm{~h}$. Spores were harvested and washed 5-10 times with cold distilled water. All spore preparations were free $(>99 \%)$ of vegetative and sporulating cells. For the germination assay, the heat-activated spores were diluted in germination buffer $(10 \mathrm{mM} \mathrm{NaCl}, 10 \mathrm{mM}$ Tris/ $\mathrm{HCl}, \mathrm{pH} 7.4)$. Small aliquots $(1.2 \mathrm{ml})$ of the heat-activated spores at an $\mathrm{OD}_{600}$ of 1 (about $1.5 \times 10^{8}$ c.f.u. $\mathrm{ml}^{-1}$ ) were supplemented with inosine (ranging from $0.01 \mathrm{mM}$ to $10 \mathrm{mM}$ ), adenosine $(0.1-2 \mathrm{mM})$ or guanosine (0.1-2 mM) separately, or $0.01 \mathrm{mM}$ inosine with $1 \mathrm{mM}$ L-alanine, and incubated at $37^{\circ} \mathrm{C}$. The decrease in $\mathrm{OD}_{600}$ was monitored for up to $60 \mathrm{~min}$ for inosine-induced germination and $90 \mathrm{~min}$ for adenosine-induced germination. Phase darkening of germinated spores was observed by phase-contrast microscopy. The data obtained are means from triplicate experiments performed with three independent spore preparations.

Expression and purification of lunH. To study the properties of IunH in vitro, it was necessary to obtain an adequate amount of IunH protein. Therefore, iun $H$ was amplified from the genomic DNA of $B$. thuringiensis with the primers P9 (5'-GGACTTCCATATGAGAATAGTTAATAAGAAAA- $3^{\prime}$, NdeI site is underlined) and P10 (5'CGGAATTCTTAAGGACAATCTGGCT-3', EcoRI site is underlined). The start codon (GTG) was replaced by ATG. The amplified fragment was digested with $\mathrm{NdeI}$ and EcoRI, and inserted into the corresponding sites of pET28a to generate a recombinant plasmid, pIE. Then, pIE was transformed into E. coli BL21(DE3) for high-level expression of iunH under the control of the T7 promoter. As the $\mathrm{N}$ terminus of IunH was designed to contain six consecutive histidines, the His6-tagged IunH was purified to homogeneity by Ni-NTA affinity chromatography. The concentration of the purified protein was determined by the method of Bradford using BSA as standard (Smith et al., 1985).

Enzyme assays. Since IunH contains one consensus $\mathrm{N}$-terminal \{D, $\mathrm{N}$ XXDXXXDD aspartate cluster which is a fingerprint for $\mathrm{NH}$ enzymes, the enzymic activity of His6-tagged IunH was measured using inosine, adenosine, guanosine, uridine or cytidine as substrate. A volume $(200 \mu \mathrm{l})$ of the reaction mixture, containing $50 \mathrm{mM}$ HEPES ( $\mathrm{pH}$ 7.3), the purified His6-tagged IunH and substrate, was incubated for $5 \mathrm{~min}$ at room temperature. Hydrolysis of the substrate (inosine, uridine or cytidine) was followed by continuous reading of $A_{280}$ on a Beckman DU-800 UV spectrophotometer. The conversion of a $1 \mathrm{mM}$ solution of inosine, uridine or cytidine to products resulted in a change in $A_{280}$ of $0.92,2.04$ and 3.42, respectively, at $\mathrm{pH} 7.3$ (Parkin et al., 1991). Hydrolysis of adenosine or guanosine was determined with the reducing sugar assay described by Parkin (1996).

The kinetic parameters $K_{\mathrm{m}}$ and $V_{\max }$ were determined at room temperature using a $1 / v-1 /[S]$ plot, where $[S]$ is the concentration of inosine (between 0.2 and $2.5 \mathrm{mM}$ ). $k_{\text {cat }}$ was derived from the equation $k_{\mathrm{cat}}=V_{\max } /[E]$, where $[E]$ is the concentration of IunH in the reaction mixture.

To check the effect of $\mathrm{pH}$, IunH activity was measured in the following buffers: $100 \mathrm{mM}$ potassium phosphate, $50 \mathrm{mM}$ HEPES, $30 \mathrm{mM}$ CHES and $30 \mathrm{mM}$ MES, covering the $\mathrm{pH}$ range from 4 to 10 . To assess the optimal temperature of the enzyme, the inosine hydrolase activity of IunH was assayed at temperatures ranging from 40 to $100{ }^{\circ} \mathrm{C}$.

Inosine hydrolase activity of the intact spores and the vegetative cells. Inosine hydrolase activity in the intact spores or the vegetative cells was determined spectrophotometrically using the difference in absorption between the nucleoside and the purine base (Parkin, 1996). All measurements were carried out at room temperature. Intact spores or vegetative cells at an $\mathrm{OD}_{600}$ of about 1 were incubated with $0.5 \mathrm{mM}$ inosine in a total volume of $900 \mu \mathrm{lof}$ $50 \mathrm{mM}$ HEPES ( $\mathrm{pH} \mathrm{7.3)} \mathrm{for} \mathrm{0,10,20,30} \mathrm{and} 40 \mathrm{~min}$, respectively. Spores or vegetative cells were removed by centrifugation. The supernatant was assayed for a change in absorbance at $280 \mathrm{~nm}$, and the resultant pellets were dried at $42{ }^{\circ} \mathrm{C}$ for at least $24 \mathrm{~h}$ to determine their dry weight.

\section{RESULTS}

\section{Cloning of an iunH-homologous gene from B. thuringiensis}

BLAST searches of the B. cereus ATCC 14579 genomic sequence released by the DOE Joint Genome Institute (www.jgi.doe.gov) revealed five predicted proteins (GenBank accession numbers BC2331, BC2683, BC2889, $\mathrm{BC} 3552$ and BC5134) that are homologous to $\mathrm{NH}$. The amino acid sequence of BC2889 is almost identical to the amino acid sequence of the putative $\mathrm{NH}$ purified from exosporia of $B$. cereus and B. anthracis (Redmond et al., 2004; Steichen et al., 2003; Todd et al., 2003). Based on the sequence of BC2889 and its homologues found in members of the $B$. cereus group, a $1.4 \mathrm{~kb}$ DNA fragment was amplified from $B$. thuringiensis genomic DNA by PCR. Sequencing showed that the DNA fragment contains an ORF, which was designated iunH (Fig. 1). iunH consists of $966 \mathrm{nt}$ and encodes a protein containing 321 aa with a predicted molecular mass of $36.2 \mathrm{kDa}$. IunH contains one $\mathrm{N}$-terminal $\{\mathrm{D}, \mathrm{N}\}$ XDXXXDD aspartate cluster, which is a fingerprint for $\mathrm{NH}$ enzymes, and one conserved $\{\mathrm{V}, \mathrm{I}, \mathrm{L}, \mathrm{M}\} \mathrm{HD}\{\mathrm{P}, \mathrm{A}, \mathrm{L}\}$ tetrapeptide sequence approximately 230 aa downstream from the N-terminal aspartate cluster (Giabbai \& Degano, 2004) (Fig. 2).

\section{Disruption of iunH increases the rate of spore germination initiated by inosine or adenosine}

To investigate the physiological role of IunH in $B$. thuringiensis, an iun $H$ disruption mutant of $B$. thuringiensis was constructed by homologous recombination (Fig. 1). The iunH disruption mutant showed normal colony morphology, growth and sporulation. Spore germination was measured by monitoring the decrease in $\mathrm{OD}_{600}$ of a spore suspension at $37{ }^{\circ} \mathrm{C}$ after the addition of either 


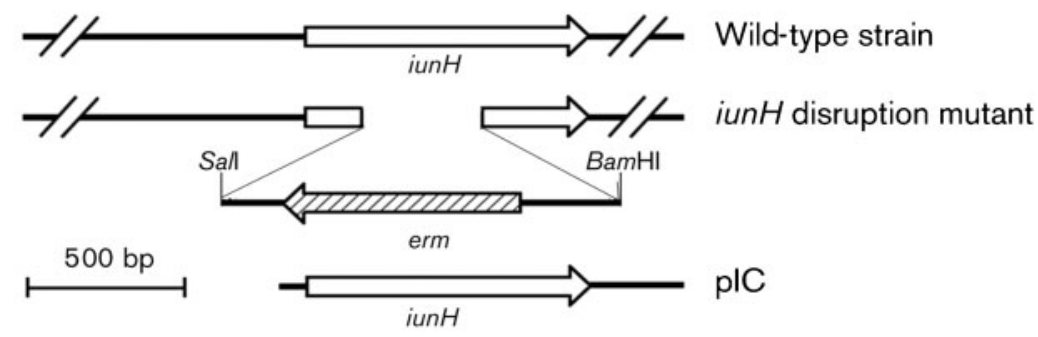

Fig. 1. Disruption of the iunH gene in $B$. thuringiensis. The arrow represents iun $H$ and its orientation, and the figure is to scale. The iunH gene was interrupted by an erythromycinresistant cassette (erm) in the disruption mutant. The entire iunH gene with its promoter was inserted into pKSV7 to generate pIC.

inosine or adenosine at the desired concentrations to the heat-activated spores. The dependence of spore germination on inosine concentration was measured in the wildtype strain, the iunH disruption mutant and the complemented strain. The germination rates of all spores were increased when the concentration of inosine was increased from 0.01 to $1 \mathrm{mM}$. As the germination rate was low when spores were induced by $0.01 \mathrm{mM}$ inosine, no significant

(a)

lunH B. thuringiensis

lunH B. cereus

lunH B. anthracis

lunH L. major

lunH C. fasciculata

URH1 S. cerevisiae

YeiK E. coli

IAG-NH T. brucei brucei

$\mathrm{NH}$ A. aegypti

$\mathrm{NH} X$. laevis

$\mathrm{NH} D$. melanogaster

(b)

lunH B. thuringiensis

lunH B. cereus

lunH $B$. anthracis

lunH L. major

lunH C. fasciculata

URH1 S. cerevisiae

YeiK E. coli

IAG-NH T. brucei brucei

NH A. aegypti

$\mathrm{NH} X$. laevis

NH D. melanogaster difference could be observed among the three strains (Fig. 3a). When $0.1 \mathrm{mM}$ inosine was used, the $\mathrm{OD}_{600}$ of the spore suspension of the iun $H$ disruption mutant decreased by about $45 \%$ during germination in contrast to a decrease of about $20 \%$ in the wild-type spore suspension. As expected, the $\mathrm{OD}_{600}$ of the complemented strain spores
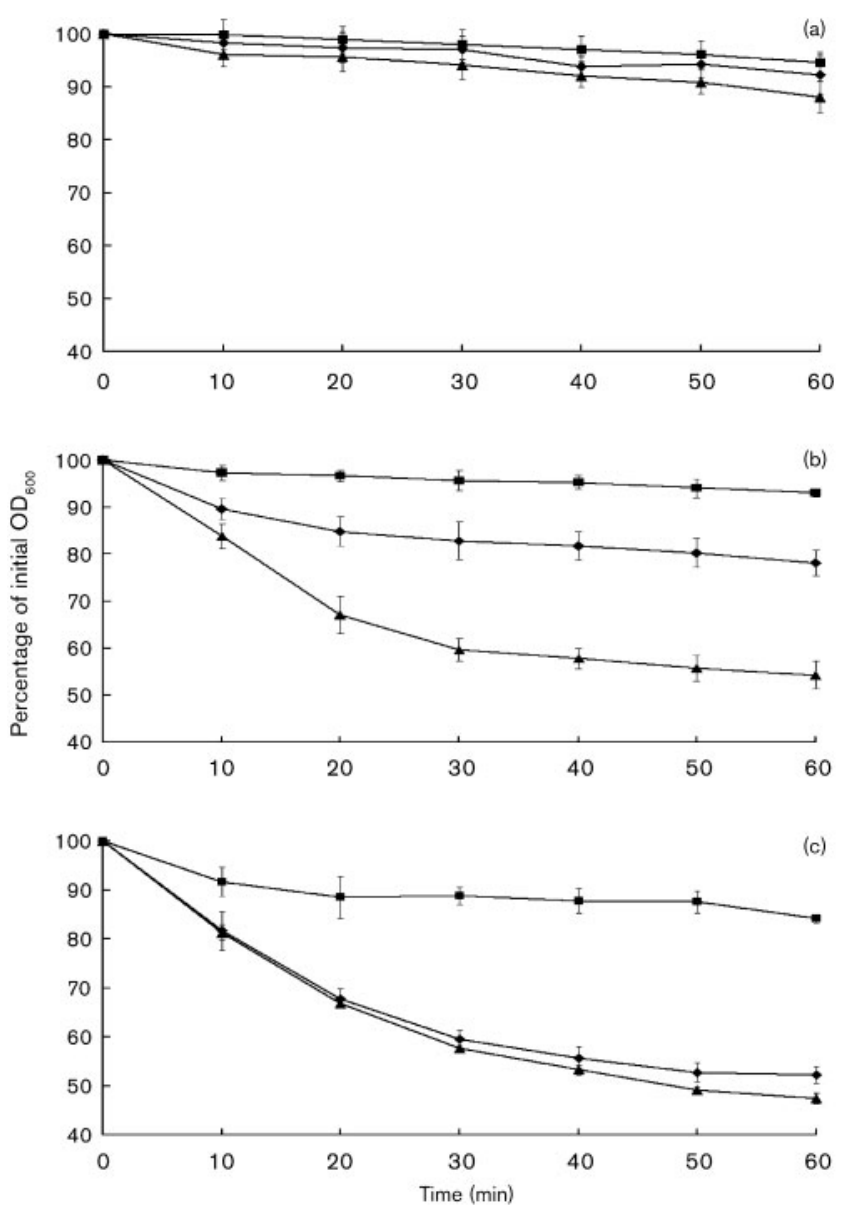

Fig. 3. Effect of iunH on spore germination initiated by inosine in $B$. thuringiensis. Spores of the wild-type $(\boldsymbol{)})$, the iunH disruption mutant $(\boldsymbol{A})$ or the complemented strain $(\boldsymbol{\square})$ were heat-activated and subsequently incubated in germination buffer supplemented with 0.01 (a), 0.1 (b) or $1 \mathrm{mM}$ (c) inosine. The decrease in $\mathrm{OD}_{600}$ was measured periodically and plotted as a percentage of the initial $\mathrm{OD}_{600}$ versus time. 


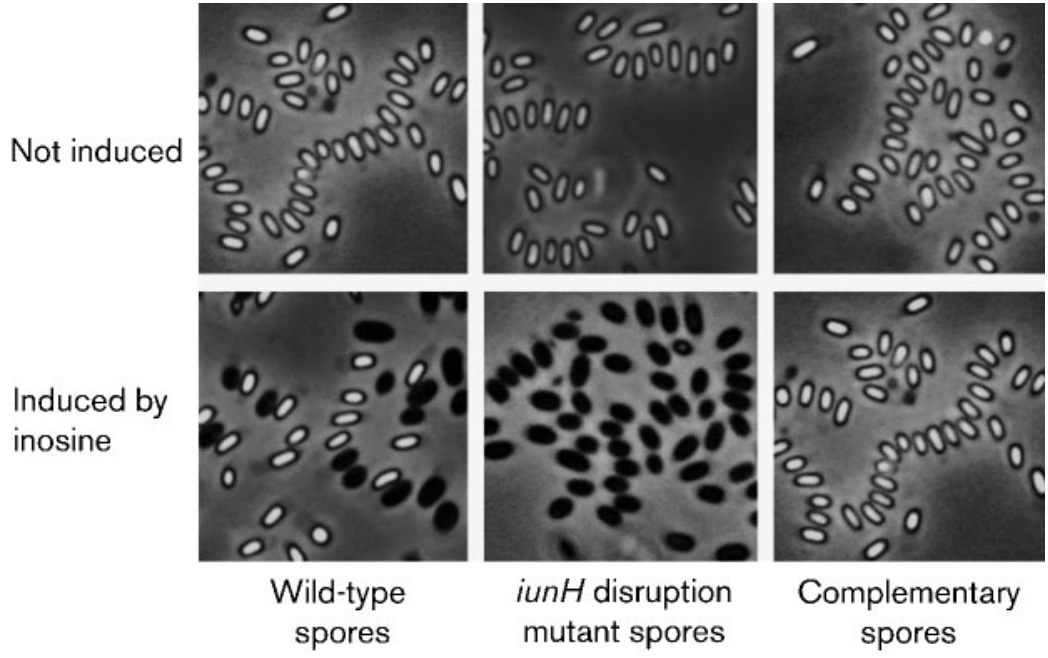

Fig. 4. Nucleoside-induced, germinationassociated, phase bright-phase dark transition efficiency in $B$. thuringiensis. All the iunH mutant spores became completely phase-dark after incubation with $0.1 \mathrm{mM}$ inosine for 30 min, while only some of the wild-type spores and almost none of the complemented strain spores turned phase-dark under these conditions. decreased by less than $10 \%$, which is even less than that of the wild-type spores (Fig. 3b). Phase-contrast microscopy showed that all the iun $H$ mutant spores became completely phase-dark after incubation with $0.1 \mathrm{mM}$ inosine for $30 \mathrm{~min}$, while only some of the wild-type spores and almost none of the complemented strain spores turned phase-dark under these conditions (Fig. 4). When the concentration of inosine was increased to $1 \mathrm{mM}$, the germination rates of both the iun $H$ disruption mutant and the wild-type spores increased significantly. However, the germination rate of complemented strain spores increased only slightly (Fig. 3c) and complemented strain spores could only germinate completely at a final concentration of $10 \mathrm{mM}$ inosine (data not shown). L-Alanine $(1 \mathrm{mM})$ could not induce spore germination, but concomitant addition of $0.01 \mathrm{mM}$ inosine could stimulate the germination in the wild-type strain and in the iun $H$ disruption mutant, while the germination rate of complemented strain spores did not increase (data not shown).

In addition to inosine, adenosine has been found to be required for spore germination of several Bacillus species (Hills, 1949; Lawrence, 1955; Hornstra et al., 2006). Adenosine-induced germination of wild-type spores, the iun $H$ mutant spores and the complemented strain spores was assayed in $B$. thuringiensis. As with inosine, the germination rate increased with increasing concentrations of adenosine. When $0.5 \mathrm{mM}$ adenosine was used, the $\mathrm{OD}_{600}$ of the $i u n H$ disruption mutant spore suspension decreased by about $50 \%$ during germination in contrast to a decrease of about $30 \%$ in the wild-type spore suspension. However, the $\mathrm{OD}_{600}$ of the complemented strain spore suspension decreased by only about $10 \%$ (Fig. 5). Only a small percentage of complemented strain spores could germinate (date not shown) even at the highest concentration of adenosine used (about $3 \mathrm{mM}$, due to the low solubility of adenosine). In addition, guanosine, which is also a substrate of IunH, barely induced spore germination. Therefore, iunH can moderate the inosine- or adenosineinduced spore germination rate in $B$. thuringiensis.

\section{Substrate specificity of IunH}

To characterize its function and substrate specificity, iun $H$ was overexpressed in E. coli BL21(DE3). Although the bulk of the expressed protein was in inclusion bodies, a clear band of IunH was observed in the supernatant of the extracts of E. coli BL21(DE3)/pIE (Fig. 6). His6-tagged IunH was purified to homogeneity by Ni-NTA affinity chromatography and the enzyme activity of IunH was measured with inosine, adenosine, guanosine, uridine or cytidine as substrate. As shown in Table 2, IunH can hydrolyse inosine, adenosine and guanosine with the following activity order: inosine $>$ adenosine $>$ guanosine. The $K_{\mathrm{m}}$ and $K_{\text {cat }}$ values with adenosine or guanosine as substrate were not calculated due to the low sensitivity of the method employed for assaying reducing sugars. Hydrolase activity could not be detected when uridine or cytidine was used as a substrate. Thus, IunH belongs to a

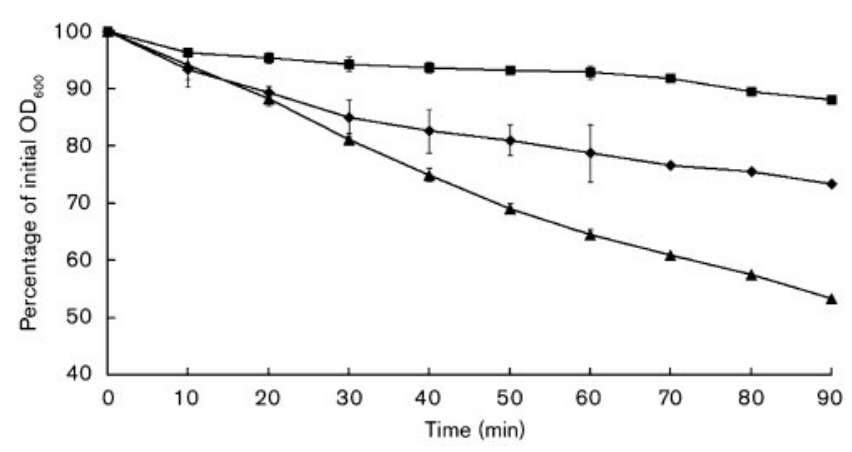

Fig. 5. Effect of iunH on spore germination initiated by $0.5 \mathrm{mM}$ adenosine in $B$. thuringiensis. Spores of the wild-type $(\boldsymbol{\nabla})$, the iun $\mathrm{H}$ disruption mutant $(\boldsymbol{\Lambda})$ or the complemented strain $(\boldsymbol{\square})$ were heat-activated and subsequently incubated in germination buffer supplemented with $0.5 \mathrm{mM}$ adenosine. The decrease in $\mathrm{OD}_{600}$ was measured periodically and plotted as a percentage of the initial $O_{600}$ versus time. 


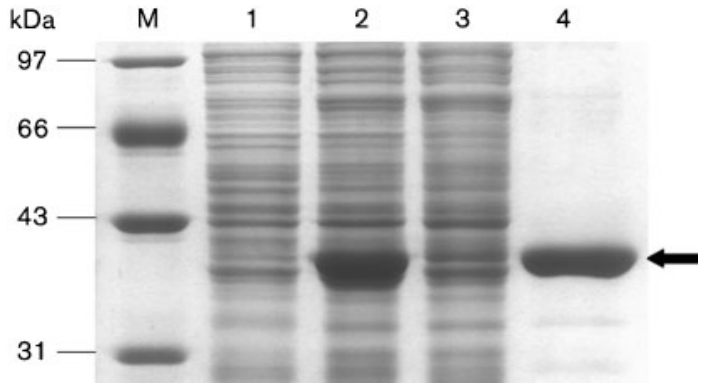

Fig. 6. SDS-PAGE of lunH expressed in $E$. coli and its purification. Lanes: $M$, standard molecular mass markers; 1 , total protein of IPTG-uninduced BL21(DE3) containing plE; 2, total protein of IPTG-induced BL21(DE3) containing pIE; 3, supernatant of IPTG-induced BL21(DE3) containing pIE; 4, purified lunH protein from Ni-NTA affinity chromatography. Samples were separated on an $8 \%$ SDS-PAGE gel and stained with Coomassie brilliant blue R-250. The arrow indicates the position of lunH.

class of purine-specific NHs (inosine/adenosine/guanosinepreferring $\mathrm{NH}$ ), based on its substrate specificity.

\section{Kinetic characterization of the recombinant lunH}

Kinetic studies were used to substantiate the catalytic properties of the expressed recombinant IunH. In standard reaction buffer (50 mM HEPES, pH 7.3) with inosine as substrate, the specific activity of the inosine hydrolase of IunH was $57.2 \pm 2.56 \mu \mathrm{mol} \mathrm{min}^{-1}(\mathrm{mg} \text { protein })^{-1}$. The kinetic parameters of IunH were $K_{\mathrm{m}}=399 \pm 115 \mu \mathrm{M}$, $k_{\text {cat }}=48.9 \pm 8.5 \mathrm{~s}^{-1}$ and $k_{\text {cat }} / K_{\mathrm{m}}=1.23 \times 10^{5} \mathrm{M}^{-1} \mathrm{~s}^{-1}$.

\section{The optimal pH and temperature for lunH}

The effect of $\mathrm{pH}$ on the inosine hydrolase activity of IunH was examined at room temperature with $3 \mathrm{mM}$ inosine as substrate. Inosine hydrolase activity of the purified recombinant IunH could be detected over a wide range of $\mathrm{pH}$ values, the highest activity being obtained at $\mathrm{pH} 6$ (Fig. 7a). The activity was stable over a wide temperature range, the highest activity being at $80{ }^{\circ} \mathrm{C}$ with high activity being maintained up to $100{ }^{\circ} \mathrm{C}$ (Fig. 7b). This result indicates that IunH is a highly heat-stable enzyme.

Table 2. Specific activity of lunH with different substrates

\begin{tabular}{|lc|}
\hline Substrate & Specific activity $\left[\boldsymbol{\mu} \mathbf{m o l} \mathbf{~ m i n}^{-\mathbf{1}}(\mathbf{m g} \text { protein })^{-\mathbf{1}}\right]$ \\
\hline Inosine & $57.2 \pm 2.56$ \\
Adenosine & $11.76 \pm 3.02$ \\
Guanosine & $1.32 \pm 0.44$ \\
Uridine & $<0.2$ \\
Cytidine & $<0.1$ \\
\hline
\end{tabular}

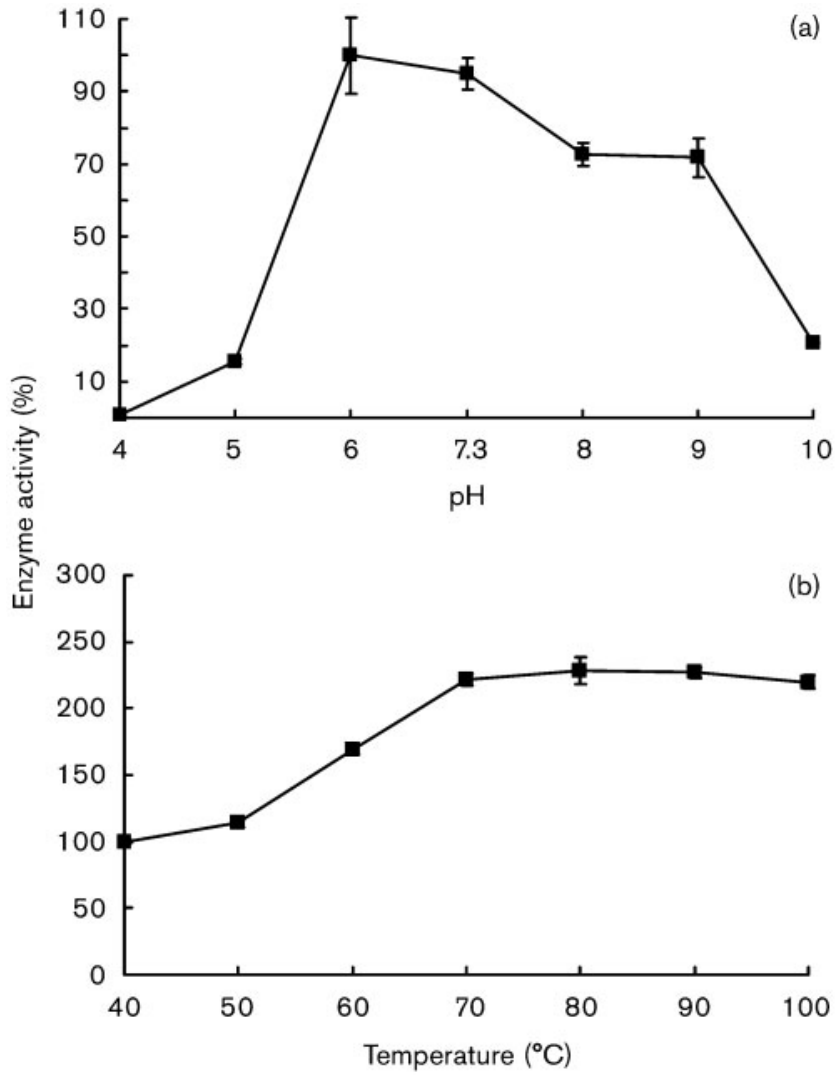

Fig. 7. Activity of purified lunH as a function of $\mathrm{pH}$ (a) and temperature (b). The inosine hydrolase activity of lunH was measured at room temperature in $100 \mathrm{mM}$ potassium phosphate, $50 \mathrm{mM}$ HEPES, $30 \mathrm{mM}$ CHES or $30 \mathrm{mM}$ MES, covering the $\mathrm{pH}$ range from 4 to 10 . To assess the optimal temperature of the enzyme, the inosine hydrolase activity of lunH was assayed at temperatures ranging from 40 to $100^{\circ} \mathrm{C}$. The activity of lunH at $\mathrm{pH} 6.0$ or at $40{ }^{\circ} \mathrm{C}$ was defined as $100 \%$.

\section{Activity of inosine hydrolase in dormant spores}

Sequence comparisons have revealed that B. thuringiensis IunH has a high level of similarity with its homologues purified from the exosporia of B. cereus and B. anthracis (Redmond et al., 2004; Steichen et al., 2003; Todd et al., 2003). IunH is probably located in the exosporium of $B$. thuringiensis spores and, therefore, inosine hydrolase activity in intact spores was measured. The specific activity of inosine hydrolase in wild-type spores of $B$. thuringiensis was $2.89 \pm 0.23 \times 10^{-2} \mu \mathrm{mol} \mathrm{min}^{-1}$ (mg dry wt) ${ }^{-1}$, while the iun $H$ mutant spores exhibited no detectable activity. The specific activity in the complemented strain spores was $8.40 \pm 0.69 \times 10^{-2} \mu \mathrm{mol} \mathrm{min}^{-1}(\mathrm{mg} \text { dry wt })^{-1}$, which is almost threefold higher than observed in the wild-type spores (Fig. 8). The higher activity of IunH in complemented strain spores may be due to the introduction of multiple copies of iunH by pKSV7 (Smith \& Youngman, 1992). Although there was considerable IunH activity in the spores, no activity was detected in intact vegetative cells 


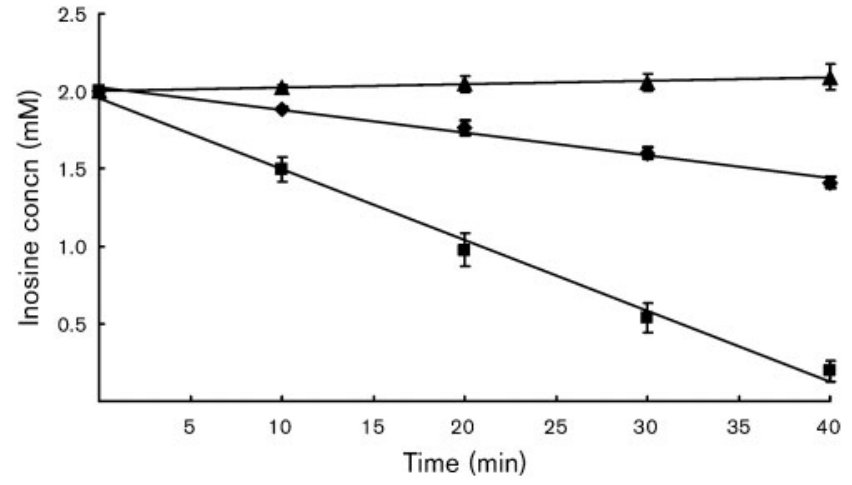

Fig. 8. Activity of inosine hydrolase in whole, dormant spores. Whole spores of the wild-type $(\boldsymbol{)})$, the iun $\mathrm{H}$ disruption mutant $(\boldsymbol{\bullet})$ or the complemented strain ( $\boldsymbol{\square})$ were incubated with $0.5 \mathrm{mM}$ inosine in a total volume of $900 \mu 50 \mathrm{mM}$ HEPES ( $\mathrm{pH}$ 7.3) for 0 , $10,20,30$ and $40 \mathrm{~min}$, respectively. Spores were removed by centrifugation and the supernatant was assayed for the change in $\mathrm{OD}_{280}$. Spore preparations whose optical density was examined in advance, were centrifuged and the deposits were dried at $42{ }^{\circ} \mathrm{C}$ for at least $24 \mathrm{~h}$ to determine their dry weight.

from the wild-type, iunH disruption mutant and complemented strains (data not shown).

\section{DISCUSSION}

Inosine-initiated spore germination starts with the interaction of inosine with its corresponding receptors, such as GerI, GerQ and GerR. Then the spores undergo the release of dipicolinic acid and cations, hydrolysis of the peptidoglycan cortex, germ-cell-wall expansion and finally resumption of vegetative growth (Hornstra et al., 2006; Setlow, 2003). This process has been described in detail, but how it is regulated is still poorly understood. Here, we provide evidence that the amount of inosine or adenosine acting as germinant can be modulated by the activity of the inosine hydrolase IunH, which converts inosine to hypoxanthine and ribose, and adenosine to adenine and ribose. This allows for modulation of the inosine- or adenosine-induced germination efficiency. Disruption of iun $H$ indeed resulted in an increase in the inosine- or adenosine-induced germination rate, whereas overexpression in the complemented strain resulted in a significant decrease of its germination-triggering capacity.

Unlike the NH from $C$. fasciculata, whose activity decreases rapidly at $\mathrm{pH}$ values below 7 (Parkin et al., 1991), B. thuringiensis IunH showed highest inosine hydrolase activity at $\mathrm{pH} 6$. Given the fact that the spores can germinate in the alkaline environment of the insect gut (Schnepf et al., 1998), the relatively low activity of IunH at alkaline $\mathrm{pH}$ values may increase the sensitivity of spores to the germinant. In addition, the heat stability of $\operatorname{IunH}$ enables it to function even after the spores have been exposed to high temperatures, which is especially important when dealing with spores in feeds and foods.

The in vivo inosine hydrolase activity indicates that the spores are able to monitor inosine levels in their environment. The difference in inosine hydrolase activity of wild-type, disruption mutant and complemented strain spores has confirmed the role of the inosine hydrolase function of IunH in modulating inosine-induced germination. Five ORFs encoding putative inosine-preferring $\mathrm{NHs}$ have been found in the genomes of B. cereus strain ATCC 14579 and four ORFs have been found in B. cereus strain ATCC 10987 (Ivanova et al., 2003; Rasko et al., 2004). This number is similar to that found in the genome of $B$. thuringiensis (Challacombe et al., 2007). The disruption of iunH eliminates inosine hydrolase activity in spores and promotes inosine-initiated germination of $B$. thuringiensis. This indicates that IunH is the most prominent $\mathrm{NH}$ in the exosporium, moderating nucleoside-induced germination capacity in B. thuringiensis.

\section{ACKNOWLEDGEMENTS}

We are grateful to Professor Dafang Huang (Biotech Institute, CAAS, China) for the gift of plasmid pHT3101. We are also grateful to Dr Paul Babitzke (Penn State University, PA, USA) for plasmid pKSV7. This work was supported by grants from the National Natural Science Foundation of China (Grant Nos. 30430010) and the National Basic research Program of China.

\section{REFERENCES}

Boydston, J. A., Yue, L., Kearney, J. F. \& Turnbough, C. L., Jr (2006). The ExsY protein is required for complete formation of the exosporium of Bacillus anthracis. J Bacteriol 188, 7440-7448.

Challacombe, J. F., Altherr, M. R., Xie, G., Bhotika, S. S., Brown, N., Bruce, D., Campbell, C. S., Campbell, M. L., Chen, J. \& other authors (2007). The complete genome sequence of Bacillus thuringiensis $\mathrm{Al}$ Hakam. J Bacteriol 189, 3680-3681.

Crickmore, N. (2006). Beyond the spore - past and future developments of Bacillus thuringiensis as a biopesticide. J Appl Microbiol 101, 616-619.

Foerster, H. F. \& Foster, J. W. (1966). Response of Bacillus spores to combinations of germinative compounds. J Bacteriol 91, 1168-1177.

Giabbai, B. \& Degano, M. (2004). Crystal structure to $1.7 \AA$ of the Escherichia coli pyrimidine nucleoside hydrolase YeiK, a novel candidate for cancer gene therapy. Structure 12, 739-749.

Gonzalez, J. M., Jr, Dulmage, H. T. \& Carlton, B. C. (1981). Correlation between specific plasmids and delta-endotoxin production in Bacillus thuringiensis. Plasmid 5, 352-365.

Gopaul, D. N., Meyer, S. L., Degano, M., Sacchettini, J. C. \& Schramm, V. L. (1996). Inosine-uridine nucleoside hydrolase from Crithidia fasciculata. Genetic characterization, crystallization, and identification of histidine 241 as a catalytic site residue. Biochemistry 35, 5963-5970.

Helgason, E., Okstad, O. A., Caugant, D. A., Johansen, H. A., Fouet, A., Mock, M., Hegna, I. \& Kolsto, A. B. (2000). Bacillus anthracis, Bacillus cereus, and Bacillus thuringiensis - one species on the basis of genetic evidence. Appl Environ Microbiol 66, 2627-2630. 
Hills, G. M. (1949). Chemical factors in the germination of sporebearing aerobes; the effect of yeast extract on the germination of Bacillus anthracis and its replacement by adenosine. Biochem J 45, 353-362.

Hornstra, L. M., de Vries, Y. P., de Vos, W. M., Abee, T. \& WellsBennik, M. H. (2005). gerR, a novel ger operon involved in L-alanineand inosine-initiated germination of Bacillus cereus ATCC 14579. Appl Environ Microbiol 71, 774-781.

Hornstra, L. M., de Vries, Y. P., Wells-Bennik, M. H., de Vos, W. M. \& Abee, T. (2006). Characterization of germination receptors of Bacillus cereus ATCC 14579. Appl Environ Microbiol 72, 44-53.

Ivanova, N., Sorokin, A., Anderson, I., Galleron, N., Candelon, B., Kapatral, V., Bhattacharyya, A., Reznik, G., Mikhailova, N. \& other authors (2003). Genome sequence of Bacillus cereus and comparative analysis with Bacillus anthracis. Nature 423, 87-91.

Lawrence, N. L. (1955). The cleavage of adenosine by spores of Bacillus cereus. J Bacteriol 70, 577-582.

Lereclus, D., Arantes, O., Chaufaux, J. \& Lecadet, M. (1989). Transformation and expression of a cloned delta-endotoxin gene in Bacillus thuringiensis. FEMS Microbiol Lett 51, 211-217.

Liu, Y. B., Tabashnik, B. E., Moar, W. J. \& Smith, R. A. (1998). Synergism between Bacillus thuringiensis spores and toxins against resistant and susceptible diamondback moths (Plutella xylostella). Appl Environ Microbiol 64, 1385-1389.

Matz, L. L., Beaman, T. C. \& Gerhardt, P. (1970). Chemical composition of exosporium from spores of Bacillus cereus. J Bacteriol 101, 196-201.

Parkin, D. W. (1996). Purine-specific nucleoside $N$-ribohydrolase from Trypanosoma brucei brucei. Purification, specificity, and kinetic mechanism. J Biol Chem 271, 21713-21719.

Parkin, D. W., Horenstein, B. A., Abdulah, D. R., Estupinan, B. \& Schramm, V. L. (1991). Nucleoside hydrolase from Crithidia fasciculata. Metabolic role, purification, specificity, and kinetic mechanism. J Biol Chem 266, 20658-20665.

Pelle, R., Schramm, V. L. \& Parkin, D. W. (1998). Molecular cloning and expression of a purine-specific $N$-ribohydrolase from Trypanosoma brucei brucei. Sequence, expression, and molecular analysis. J Biol Chem 273, 2118-2126.

Ramarao, N. \& Lereclus, D. (2005). The InhA1 metalloprotease allows spores of the B. cereus group to escape macrophages. Cell Microbiol 7, 1357-1364.

Rasko, D. A., Ravel, J., Okstad, O. A., Helgason, E., Cer, R. Z., Jiang, L., Shores, K. A., Fouts, D. E., Tourasse, N. J. \& other authors (2004). The genome sequence of Bacillus cereus ATCC 10987 reveals metabolic adaptations and a large plasmid related to Bacillus anthracis pXO1. Nucleic Acids Res 32, 977-988.

Redmond, C., Baillie, L. W., Hibbs, S., Moir, A. J. \& Moir, A. (2004). Identification of proteins in the exosporium of Bacillus anthracis. Microbiology 150, 355-363.

Ribeiro, J. M. \& Valenzuela, J. G. (2003). The salivary purine nucleosidase of the mosquito, Aedes aegypti. Insect Biochem Mol Biol 33, 13-22.

Sambrook, J., Fritsch, E. F. \& Maniatis, T. (1989). Molecular Cloning: a Laboratory Manual, 2nd edn. Cold Spring Harbor, NY: Cold spring Harbor Laboratory.

Schnepf, E., Crickmore, N., Van Rie, J., Lereclus, D., Baum, J., Feitelson, J., Zeigler, D. R. \& Dean, D. H. (1998). Bacillus thuringiensis and its pesticidal crystal proteins. Microbiol Mol Biol Rev 62, 775-806.

Setlow, P. (2003). Spore germination. Curr Opin Microbiol 6, 550-556.

Smith, K. \& Youngman, P. (1992). Use of a new integrational vector to investigate compartment-specific expression of the Bacillus subtilis spoIIM gene. Biochimie 74, 705-711.

Smith, P. K., Krohn, R. I., Hermanson, G. T., Mallia, A. K., Gartner, F. H., Provenzano, M. D., Fujimoto, E. K., Goeke, N. M., Olson, B. J. \& Klenk, D. C. (1985). Measurement of protein using bicinchoninic acid. Anal Biochem 150, 76-85.

Steichen, C., Chen, P., Kearney, J. F. \& Turnbough, C. L., Jr (2003). Identification of the immunodominant protein and other proteins of the Bacillus anthracis exosporium. J Bacteriol 185, 1903-1910.

Steichen, C. T., Kearney, J. F. \& Turnbough, C. L., Jr (2005). Characterization of the exosporium basal layer protein BxpB of Bacillus anthracis. J Bacteriol 187, 5868-5876.

Stewart, B. T. \& Halvorson, H. O. (1953). Studies on the spores of aerobic bacteria. I. The occurrence of alanine racemase. J Bacteriol $\mathbf{6 5}$, 160-166.

Todd, S. J., Moir, A. J., Johnson, M. J. \& Moir, A. (2003). Genes of Bacillus cereus and Bacillus anthracis encoding proteins of the exosporium. J Bacteriol 185, 3373-3378.

Versees, W., Decanniere, K., Pelle, R., Depoorter, J., Brosens, E., Parkin, D. W. \& Steyaert, J. (2001). Structure and function of a novel purine specific nucleoside hydrolase from Trypanosoma vivax. J Mol Biol 307, 1363-1379.

Yan, X., Gai, Y., Liang, L., Liu, G. \& Tan, H. (2007). A gene encoding alanine racemase is involved in spore germination in Bacillus thuringiensis. Arch Microbiol 187, 371-378.

Edited by: T. Abee 